\title{
Synthesis problems: role of mathematical complexity in students' problem solving strategies
}

\author{
Bashirah Ibrahim ${ }^{1}$, Lin Ding ${ }^{1}$, Daniel R. White², Ryan Badeau², and Andrew F. Heckler ${ }^{2}$ \\ ${ }^{1}$ The Ohio State University, Department of Teaching and Learning, \\ 1945 N High St, Columbus, OH 43210 \\ ${ }^{2}$ The Ohio State University, Department of Physics, \\ 191 West Woodruff Ave, Columbus, OH 43210
}

\begin{abstract}
We report a study on students' approaches to quantitative synthesis problems with varying mathematical complexities. Synthesis problems involve multiple concepts typically taught in different chapters. In this study, mathematical complexity is determined by the number and the type of equations that must be simultaneously solved. Students from a second year calculus-based physics course were randomly assigned to solve one of three synthesis problems varying in mathematical complexity: simple, medium, or complex. Results from extended written and interview responses revealed four major problem-solving approaches used by the students: trial-and-error, flawed reasoning, knowledgeable, and expert-like approach. Students solving the simple problem used all the four approaches, whereas those solving the other two mainly used the "trial-and-error" or "flawed reasoning" approaches. A common phenomenon is that many students could identify the appropriate concepts but failed to correctly apply them. Additionally, the students made similar mistakes on all the three problems.
\end{abstract}

\section{INTRODUCTION}

Most of the previous work on physics problem solving has utilized single-concept problems. There is a dearth of studies on problems comprising multiple concepts which explicitly addressed this research area [1-3]. Our study is based on synthesis problems which are tasks consisting of at least two distinct concepts, typically from different chapters and separated in the teaching timeline [1]. The core of synthesis problems is the merging of multiple concepts as well as equations (emerging on concepts application) in order to build a solution. Initial studies on synthesis problem solving [1,2] have highlighted the key role of guided conceptual scaffolding to support students' recognition and application of relevant concepts. Another study identified concept recognition and application as the main bottlenecks in solving synthesis problems [3].

This paper reports on students' problem solving strategies when handling quantitative synthesis problems with varying mathematical complexity. In this study we will operationally define mathematical complexity as the number and type of equations required to be simultaneously solved. For example, consider a problem involving two equations and two unknowns. We propose that a problem in which both unknowns are in both equations is more mathematically complex than a problem in which only one unknown is in each equation. Further, we propose that a problem involving unknowns in non-linear relationships (such as a quadratic or trigonometric) is more mathematically complex than a problem involving unknowns in linear equations. Note that in this study, the physics problems themselves do not change per se, rather the variables which are known and unknown are manipulated, thus varying the mathematical complexity.

We posit that in solving quantitative synthesis problems, mathematical complexity is an additional potential bottleneck that may interfere with the students' identification and application of relevant concepts and hence their problem solving strategies. Here, problem solving strategy refers to a sequence of steps to be implemented in an attempt to solve problems [4], which aligns with our goal of providing insights into the students' abilities to identify and apply relevant concepts in tackling synthesis problems.

The following research question is addressed: What are the approaches that students use for solving quantitative synthesis physics problems with varying mathematical complexity?

\section{METHODOLOGY}

By varying which variables are known and unknown, thus varying the mathematical complexity, we designed three versions of the same problem, simple, medium and complex (see Figure 1). Each task involves a block on an inclined ramp, being propelled from a spring. The block undergoes a trajectory and lands on another inclined surface. Conservation of energy and projectile motion are the two pertinent concepts to solve the three tasks.

We acknowledge that students may employ a variety of concepts and/or equations. However, four fundamental equations (shown in Figure 2) are required to solve all three 
problems. One equation is needed for energy conservation relating the different energy sources present in the system (equation (1) in Fig. 2). Three equations are required for projectile motion. These equations correspond to horizontal (equation (2) in Fig. 2) and vertical (equation (3) in Fig. 2) motion, and the trigonometry relating the angle $\phi$, the magnitude and direction of the horizontal and vertical components of motion (equation (4) in Fig. 2).

The mass of the block (m), spring constant $(\mathrm{k})$, equilibrium position (L) and angle $\phi$ are the common information provided in all the three tasks. The simple task also provides information about the initial compression of the spring $(x)$ and the angle of initial slope $(\theta)$. It asks for the horizontal distance $(R)$. Thus, equation (1) is readily solved for $v$ since it has only one unknown. This leaves three equations, (2)-(4) in Fig. 2, with 2 unknowns, which with some relatively simple manipulation results in a linear and simple equation for $R$. The medium problem provides information about $\theta$ and $R$. It asks for $x$. The complex problem provides information about $x$ and $R$, and asks for $\theta$. Both of these problems require solving 4 equations with multiple unknowns. The complex problem is rated as more complex since it involves using the quadratic equation and trigonometric functions.

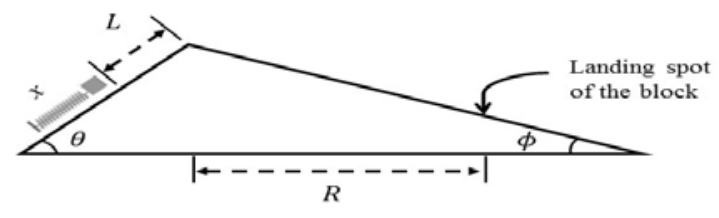

Simple: An apparatus uses a spring launcher to fire a block (mass $2 \mathrm{~kg}$ ) over a ridge. The spring (spring constant, $\mathrm{k}=3000 \mathrm{~N} / \mathrm{m}$ ) is compressed $0.1 \mathrm{~m}$ and releases the block from its equilibrium position, which is located a distance $\mathrm{L}=0.5 \mathrm{~m}$ from the crest. If the angles shown are $\theta=35^{\circ}$ and $\phi=25^{\circ}$, what is the horizontal distance $R$ from the crest to where the block hits the slope on the other side?

Medium: An apparatus uses a spring launcher to fire a block (mass $2 \mathrm{~kg}$ ) over a ridge. The spring (spring constant, $\mathrm{k}=3000 \mathrm{~N} / \mathrm{m}$ ) is compressed an unknown distance $x$, and releases the block from its equilibrium position, which is located a distance $\mathrm{L}=0.5 \mathrm{~m}$ from the crest. The block lands on the opposite slope, a horizontal distance $R=1.3 \mathrm{~m}$ from the crest. If the angles shown are $\theta=35^{\circ}$ and $\phi=25^{\circ}$, what was the initial compression of the spring, $x$ ?

Complex: An apparatus uses a spring launcher to fire a block (mass $2 \mathrm{~kg}$ ) over a ridge. The spring (spring constant, $\mathrm{k}=3000 \mathrm{~N} / \mathrm{m}$ ) is compressed $0.1 \mathrm{~m}$ and releases the block from its equilibrium position, which is located a distance $\mathrm{L}=0.5 \mathrm{~m}$ from the crest. The block lands on the opposite side, a horizontal distance $R=1.3 \mathrm{~m}$ from the crest. If $\phi=25^{\circ}$, what was the angle of the initial slope, $\Theta$ ?

FIG. 1. The three versions of the synthesis problems.

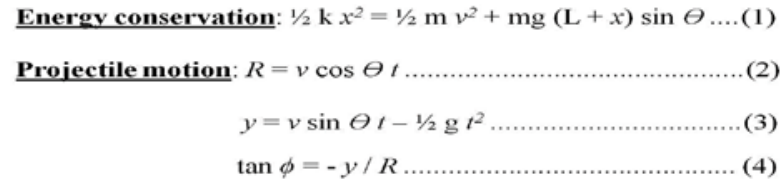

Our sample consists of 13 students, enrolled in a second year mechanics course for physics majors. These students were often exposed to synthesis problems in their mechanics course. Each student participated in a 50-minute individual interview session and was randomly assigned to either the simple $(n=4)$, medium $(n=5)$, or complex $(n=$ 4) problem. In each session, the student was initially allocated 20-25 minutes to solve the problem. The interview immediately followed, lasting a maximum of 25 minutes.

From the written responses, we coded the concepts that the students identified, and how (correctly or incorrectly) they applied the two pertinent concepts. We captured the conceptual errors made when the pertinent concepts were incorrectly applied. We also considered whether the students combined the energy conservation and projectile motion equations to find a value or an expression for the unknown. Finally, we coded the potential cause (mathematical or conceptual errors or a combination) for an incorrect solution. The interview responses were analyzed thematically for the following: (i) factors cueing the identification of particular concepts, and (ii) reasons for incorrect application of pertinent concepts.

We constructed "profiles" based on the students' written and interview responses. The profiles provide a holistic description of the problem solving approaches by considering the following three themes: (i) concept identification, (ii) concept application and, (iii) synthesis of energy conservation and projectile motion equations. For each student, the written solution and interview responses were considered together, summarized and described thus leading to a profile. The holistic descriptions (i.e profiles) are structured such that they capture the main variations in the actions and reasoning of individual students within the whole sample. This process was repeated for all the 13 students whereby the descriptors were refined. Four main profiles emerged from the data. They describe four different strategies with increasing level of sophistication for handling the synthesis problems. These are "Trial and Error", "Flawed Reasoning”, "Knowledgeable" and "Expert-like" approach. The process of profile allocation was repeated independently by two researchers and an inter-reliability rate of $86 \%$ was obtained.

\section{RESULTS}

Table 1 presents the profiles for the problem solving strategies with increasing level of sophistication. Most students (61\%) used the "Flawed Reasoning" approach. Around 23\% of the students employed the "Trial and Error" strategy. An equal percentage (8\%) of students had a "Knowledgeable" and an "Expert-like" approach respectively. Table 2 gives the distribution of students, across the four profiles for problem solving strategies, with respect to the task's mathematical complexity. Students tackling the simple task used all four approaches. Those who solved the medium and complex task only employed the "Trial and Error" or "Flawed Reasoning" strategy. 
TABLE 1. Profiles for strategies used to handle the synthesis problems $(\mathrm{n}=13)$.

\begin{tabular}{|c|c|c|c|c|}
\hline & \multicolumn{4}{|l|}{ Types of Problem $\mathrm{S}$} \\
\hline Themes & Trial and Error & Flawed Reasoning & Knowledgeable & Expert-like \\
\hline $\begin{array}{c}\text { Concept } \\
\text { identification }\end{array}$ & $\begin{array}{l}\text { Initially identify Newton"s } \\
\text { Second Law, cued by the need } \\
\text { to use constant acceleration } \\
\text { equations for } 2 D \text { motion aspect } \\
\text { of task. Newton"s Second Law } \\
\text { yields acceleration from which } \\
\text { velocity is obtained. However, } \\
\text { energy conservation is invoked } \\
\text { when there is failure to find } \\
\text { velocity. }\end{array}$ & \multicolumn{3}{|c|}{$\begin{array}{l}\text { Identify energy conservation and projectile motion at the } \\
\text { onset. Energy conservation is cued by (i) the spring being the } \\
\text { source for velocity (ii) the change in heights indicating } \\
\text { presence of gravitational potential energy and (iii) familiarity } \\
\text { with similar problem. Projectile motion is cued by the object } \\
\text { having a trajectory. }\end{array}$} \\
\hline $\begin{array}{l}\text { Concept } \\
\text { application. }\end{array}$ & \multicolumn{2}{|c|}{$\begin{array}{l}\text { Incorrectly apply energy conservation and projectile } \\
\text { motion. The former involves (i) ignoring the block's } \\
\text { kinetic energy (ii) ignoring the block's gravitational } \\
\text { potential energy due to what students believe a negligible } \\
\text { change in heights (iii) incorrect expression for spring } \\
\text { potential energy, and (iv) incorrect height expression for } \\
\text { gravitational potential energy. The latter includes (i) } \\
\text { incorrectly relating the magnitude and direction of the } \\
\text { variables for trigonometry and (ii) incorrect expression } \\
\text { for initial positions in the equation for horizontal and/or } \\
\text { vertical motion. }\end{array}$} & $\begin{array}{l}\text { Incorrectly apply } \\
\text { energy } \\
\text { conservation with } \\
\text { absence of } \\
\text { gravitational } \\
\text { potential energy. } \\
\text { Correctly apply } \\
\text { projectile motion. }\end{array}$ & $\begin{array}{l}\text { Correctly } \\
\text { apply energy } \\
\text { conservation } \\
\text { and projectile } \\
\text { motion. }\end{array}$ \\
\hline $\begin{array}{l}\text { Synthesis of } \\
\text { energy } \\
\text { conservation } \\
\text { and } \\
\text { projectile } \\
\text { motion } \\
\text { equations }\end{array}$ & $\begin{array}{l}\text { No combination of energy } \\
\text { conservation equation with } \\
\text { projectile motion ones. The } \\
\text { task is left incomplete. }\end{array}$ & $\begin{array}{l}\text { Energy conservation } \\
\text { and projectile motion } \\
\text { equations may be } \\
\text { combined. When } \\
\text { combined: (i) an } \\
\text { incorrect value is } \\
\text { obtained due to either } \\
\text { conceptual mistakes } \\
\text { only or conceptual and } \\
\text { mathematical mistakes, } \\
\text { (ii) an incorrect } \\
\text { expression, without } \\
\text { isolating the unknown, } \\
\text { is obtained. }\end{array}$ & $\begin{array}{l}\text { Combine energy } \\
\text { conservation and } \\
\text { projectile motion } \\
\text { equations. The } \\
\text { task is evaluated } \\
\text { but an incorrect } \\
\text { value is obtained } \\
\text { due to conceptual } \\
\text { and mathematical } \\
\text { mistakes } \\
\text { associated with } \\
\text { energy } \\
\text { conservation only. }\end{array}$ & $\begin{array}{l}\text { Combine } \\
\text { energy } \\
\text { conservation } \\
\text { and projectile } \\
\text { motion } \\
\text { equations. A } \\
\text { correct } \\
\text { expression is } \\
\text { obtained with } \\
\text { the unknown } \\
\text { isolated but } \\
\text { not } \\
\text { evaluated. }\end{array}$ \\
\hline $\begin{array}{l}\text { Percentage } \\
\text { of students }\end{array}$ & $3(23 \%)$ & $8(61 \%)$ & $1(8 \%)$ & $1(8 \%)$ \\
\hline
\end{tabular}

TABLE 2. Percentage of students with respect to task's mathematical complexity and problem solving approaches (n=13).

\begin{tabular}{|c|c|c|c|c|}
\cline { 2 - 5 } \multicolumn{1}{c|}{} & \multicolumn{4}{c|}{ Types of Problem Solving Approach } \\
\hline Task complexity & Trial and Error & Flawed Reasoning & Knowledgeable & Expert-like \\
\hline Simple & $8 \%$ & $8 \%$ & $8 \%$ & $8 \%$ \\
\hline Medium & $8 \%$ & $30 \%$ & $0 \%$ & $0 \%$ \\
\hline Complex & $8 \%$ & $22 \%$ & $0 \%$ & $0 \%$ \\
\hline
\end{tabular}

For concept identification, students from the "Trial and Error” approach initially considered Newton's Second Law cued by the use of constant acceleration equations for the $2 \mathrm{D}$ motion aspect of the problem. Hence acceleration is obtained from Newton's Second Law allowing velocity to be found. However, the students shifted to energy conservation when they failed to find a value/expression for velocity from acceleration. The following quote typifies this description: "I knew at this point [showing the 2D motion aspect] I'll be using constant acceleration equations. When I saw that we needed acceleration, I guess you can solve for acceleration using force. If I solve for acceleration [...] I really don't know how to convert it into velocity." In contrast, students from the "Flawed Reasoning", "Knowledgeable" and "Expert-like" approach identified energy conservation and projectile motion at the onset. Individual interviews revealed that the factors cueing the pertinent concepts are familiarity with similar singleconcept problems and surface features of the task.

For concept application, students from the "Trial and Error" and "Flawed Reasoning" approach incorrectly applied energy conservation and projectile motion. For the "Knowledgeable" approach, only projectile motion was correctly applied. For the "Expert-like" approach both pertinent concepts were correctly applied. The following are some typical errors for energy conservation and projectile motion respectively.

$$
\begin{aligned}
\because i, m v^{2} & =k x^{2} \\
x & =\frac{1}{2 k} m U^{2} \\
x & =U \sqrt{\frac{m}{2 k}}
\end{aligned} \quad \sum_{B}=\frac{1}{2} k\left(x_{s}\right)^{2}+m v_{i}^{2}+m g(0)
$$

FIG. 3. Conceptual errors for energy conservation.

In Figure 3(a) the student omitted the block's gravitational potential energy and used an incorrect expression for the spring potential energy. The main reason for ignoring gravitational potential energy is that there is a negligible change in height along the ramp, as shown by the quote: "I didn't even think that there would be a change in gravitational potential energy since we are travelling very 
short distances which are negligible.” In Figure 3(b), the energy conservation equation has an incorrect height expression for the block's gravitational potential energy. The spring compression $x$ was ignored when the block reaches the crest of the ramp. For projectile motion, Figure 4(a) indicates inconsistencies in the direction of motion in the equation for vertical motion. Figure 4(b) depicts incorrect initial position in the equations for horizontal and vertical motion. It also highlights that the student incorrectly relates the angle $\phi$ to the horizontal and vertical motion.

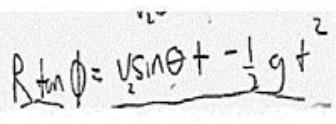

(a)

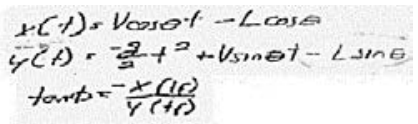

(b)
FIG. 4. Conceptual errors for projectile motion.

For synthesis of equations, students from the "Trial and Error" approach did not combine the energy conservation and projectile motion equations. Those from the "Flawed Reasoning" approach might combine the equations but they either got an incorrect value or an incorrect expression. An incorrect value was found due to either conceptual errors only or a combination of mathematical and conceptual errors. An incorrect expression was formulated due to conceptual errors only. Also, the variable to be found was often not isolated. Overall, the conceptual errors were found to be associated with both pertinent concepts. As for those in the "Knowledgeable" approach and "Expert-like" approach categories, students typically combined the equations. However, in the former case, an incorrect value was often obtained due to conceptual and mathematical errors associated with formulating and manipulating the energy conservation equation. In the latter case, even if a value was not evaluated, a final correct expression was formulated isolating the unknown to be determined.

\section{CONCLUSIONS}

Four main approaches were used when solving quantitative synthesis problems with varying mathematical complexity. Students who completed the simple task used all four strategies. Those who handled the medium and complex task mainly used the less sophisticated "Trial and Error" and "Flawed Reasoning" approach. In addition to processing the two pertinent concepts (i.e identifying, and reflecting on their applications and connections), the students have to manipulate various mathematical formalisms which became more difficult and intricate as mathematical complexity increased. Likely, the predominant use of the less sophisticated problem solving approaches to the medium and complex tasks could be due to cognitive load.

A previous study [3] identified concept recognition and application as bottlenecks for synthesis problem solving. In our case, across the three tasks, the students were able to recognize the pertinent concepts but they mostly failed to apply them appropriately. They also made similar conceptual mistakes. Thus, mathematical complexity does not seem to interact with the students' concept identification and application.

An implication of this study is that synthesis problems may promote physics problem solving as a learning tool as well as an assessment tool. In the physics context, most of the tasks are structured to use single concepts whereby students are not given the opportunity to engage deeply in identifying the most appropriate concepts, and reflecting on proper approaches to handle the problem. Single-concept problems may encourage the mechanistic identification of concepts, and the plug-and-chug approach to problem solving. In contrast, synthesis problems provide a more authentic situation to prompt qualitative task analysis. Students need to dissect the task to the core fundamentals and make decisions about the most appropriate concepts to be used such as how and why to use them. They also need to reflect on how the multiple events and concepts are connected before designing the most suitable problem solving approach. For example, in our study, we noted that students from the "Trial-and-Error" approach initially relied on Newton's Second Law to tackle the spring aspect of the problem but later shifted to energy conservation when they reached a dead-end. Further, synthesis problems cannot be solved by simple plug-and-chug. They require the blending of equations generated from applying the multiple concepts. Besides understanding the concepts underlying the equations, students need to comprehend how the different mathematical expressions are connected.

Subsequently, it is worth exploring how students tackle the mathematics of synthesis problems and the associated difficulties. In their study on conceptual scaffolding in synthesis problems, Ding et al. [1] reported that one of the reasons for the students' failure to determine the correct final value is their inability to merge multiple equations.

\section{ACKNOWLEDGEMENT}

This work is supported by the NSF (award \# DRL1252399)

[1] L. Ding, N. Reay, A. Lee and L. Bao, Phys. Rev. Phys. Educ. Res, 7, 020109 (2011).

[2] L. Ding, N. Reay, A. Heckler and L. Bao, in 2010 Physics Education Conference, edited by C. Singh, M. Sabella, and N.S. Rebello, AIP Conference Proceedings, Portland.

[3] D.R. White, R. Badeau, A.F. Heckler and L. Ding, in 2014 Physics Education Research Conference, edited by P.V. Engelhardt, A.D. Churukian, and D.L. Jones, AIP Conference Proceedings, Minneapolis, MS.

[4] J.D. Bransford and B.S. Stein, The IDEAL Problem Solver, (W.H. Freeman, New York, 1984). 